\title{
CLASSIFICATION OF ALZHEIMER USING fMRI DATA AND BRAIN NETWORK
}

\author{
Rishi Yadav, Ankit Gautam, Ravi Bhushan Mishra \\ Computer Science \& Engineering, IIT BHU (Varanasi)
}

\begin{abstract}
Since the mid of 1990s, functional connectivity study using fMRI (fcMRI) has drawn increasing attention of neuroscientists and computer scientists, since it opens a new window to explore functional network of human brain with relatively high resolution. BOLD technique provides almost accurate state of brain. Past researches prove that neuro diseases damage the brain network interaction, protein- protein interaction and gene-gene interaction. A number of neurological research paper also analyse the relationship among damaged part. By computational method especially machine learning technique we can show such classifications. In this paper we used OASIS fMRI dataset affected with Alzheimer's disease and normal patient's dataset. After proper processing the fMRI data we use the processed data to form classifier models using SVM (Support Vector Machine), KNN (K-nearest neighbour) \& Nä̈ve Bayes. We also compare the accuracy of our proposed method with existing methods. In future, we will other combinations of methods for better accuracy.
\end{abstract}

\section{KEYWORDS}

Brain Network, Dementia, Alzheimer's disease, SVM, KNN, Fmri

\section{INTRODUCTION}

Alzheimer's disease (AD), a progressive, irreversible, neurodegenerative disorder and multifaceted disease, occurs most frequently in older age. Alzheimer's disease slowly destroys brain cells causing loss of memory, thinking skill, behaviour and learning and ultimately ability to perform simple tasks. AD is the only disease among top 10 causes of death among Americans, which cannot be prevented, slowed and cured. Early diagnosis of Alzheimer's disease is very costly, time-taken and careful medical assessment of physical history, parent's health analysis, physical and neurobiological exam and Mini Mental State Examination (MMSE) etc.[13]. Therefore, development in the field of automatic computational Alzheimer's Detection is economically desirable.

Neuronal dysfunction in AD mainly causes due to failure of functional integration and damage in connectivity network of brain[6]. Brain is the centre of human nervous system. Brain is made of more than 100 billion of nerves that communicate in trillions of connections called synapses. It contains spatially distributed but functionally connected regions that continuously share stimulus and responses to each other. In past three decades, a rich study on functional and structural neuroimaging have provided a plenty of knowledge about role, functions and connectivity pattern

Dhinaharan Nagamalai et al. (Eds) : NATL, CSEA, DMDBS, Fuzzy, ITCON, NSEC, COMIT - 2018 pp. 109-119, 2018. @ CS \& IT-CSCP 2018

DOI : $10.5121 /$ csit.2018.80609 
of brain regions. A number of computational and biological tools have also developed to collect this information. [5][8][10] . As one's age passes, the brain cells develop plaques and tangles. They first developed in the areas involved in memory and then spread out to other parts of the brain. . These tangles and plaques disable or block the communication among the nerve cells and distress its function and eventually the cells will die [8].

For the early detection of Alzheimer's disease, the analysis of neuroimaging data has achieved much attraction recent years. Some neuroimaging modalities or biomarker exists in order to study and detect $\mathrm{AD}$ and other brain related diseases are positron emission tomography (PET), magnetic resonance imaging (MRI), computed tomography (CT), study of cerebral metabolism with fluoro-deoxy-d-glucose (FDG) etc. [7]. The past two decades have witnessed the increasing use of resting state functional magnetic resonance imaging (rs-fMRI) as a tool for mapping human brain network. The word 'rest' refers to a constant condition without imposed stimulus i.e. no task performance during acquisition of fMRI data. The resting brain activity is measured through observing the changes in oxygen and blood flow in the brain parts and among the brain parts, which creates a signal referred to blood-oxygen-level dependent (BOLD) signal that can be measured using functional magnetic resonance imaging technique.

The BOLD signals represents low-frequency spontaneous fluctuations of oxygen and blood flow in brain network regions. Initially it had been thought to be a noise. In Biswal et al., [14] demonstrated that that these low frequencies $(0.1$ to $0.01 \mathrm{~Hz})$ in BOLD signal are highly correlated among the brain regions. Later the low frequency fluctuations were shown to be of neural origin and specific to grey matter across the hemispheres in the bilateral motor cortices [17]. Thus the correlation structure of these rs-fMRI can be used to determine the network within the brain.

Zhang et al., [6] investigated the functional connectivity in the resting brain network by comparing the samples of healthy volunteer and patient affected with different levels of Alzheimer disease. To detect the alteration in brain network in general and posterior cingulate cortex (PCC) in particular, resting state functional magnetic resonance imaging used by the comparing of fMRI dataset. Data acquisition was performed following all the ethical and scientific protocols prescribed by Diagnostic and Statistical Manual of Mental Disorders. The study observed that the set of regions like the hippocampus, the inferior temporal cortex, the visual cortices and especially the presumes and cuneus, the medial prefrontal cortex having high degree of dissociated functional connectivity with PCC in all AD patients. Study also observed that the degree of connectivity level also intensified as the stage of AD progression increased from mild to moderate and then severe AD. Thus study concluded that alteration in functional connectivity in brain network might play a role in early diagnosis of AD.

Prasad et al.,[8] compared a variety of anatomic connectivity measures, including several novel ones like global efficiency, transitivity, path length, modularity, local efficiency, optimal community structure, eigenvector centrality etc. that may help in distinguishing Alzheimer's disease(AD) patients. They evaluated two kinds of connectivity measures. The first evaluated measures from whole-brain tractography connectivity matrices and second one studied additional network measures based on a novel flow-based measure of connectivity matrices. The study evaluated the measures' ability to discriminate disease by using 10 -fold cross-validated classifier that were repeated 30 times and found the highest accuracy of $78.2 \%$. 
Biju K Sa et al.,[1] provided the software solution of for detecting the Alzheimer's disease and brain abnormalities as well and produced a 3D representation of MRI slices. The study collected and analyzed various parameters from MRI dataset like cortex area, grey matter volume, white matter volume, and cavity area and brain density. They used grey to white matter ratio for determining if person is affected by Alzheimer's disease. MRI slices undergo with different processes like segmentation, calculation of density and volume of brain parts, de-noising and 3D construction. Finally the study concluded that the grey matter to white matter volume ratio will be more for the person having brain abnormalities. This study included brain abnormalities such as tumour and internal blood clotting etc. too.

Sarraf et al.,[2] used convolutional neural network (CNN) to classify Alzheimer brain and normal healthy brain. They used MRI slices of Alzheimer patient and normal healthy brain from ADNI databank and pre-processed the image using the standard modules of FMRIB library v5.0. Images were labelled for binary classification of Alzheimer's Vs normal dataset and these labelled images were converted to Imdb storage databases for higher throughput to be fed into Deep learning platform. The study used CNN and famous architecture LeNet-5 and successfully classified fMRI data of Alzheimer's patient from normal controls. The accuracy of test data on training data reached $96.85 \%$, which was trained and tested with huge number of images. This study also showed a novel path to use more complicated computational architecture and classifiers to increase the efficient and effective pre-clinical diagnosis of AD.

F. Previtali et al..[7]Extracted the key features from magnetic resonance imaging which are most suitable for classification task. For extraction of key features the study proposed a novel feature extraction technique that is based on recent computer vision method, called Oriented FAST and Rotated BRIEF. They used the state-of-the-art approaches on two medical established databank ADNI and OASIS. The extracted features are processed with the combination of two new metrics i.e., their spatial position and their distribution around the patient's brain, and given as input to a function-based classifier, Support Vector Machines (SVM). The study obtained a classification accuracy and sensitivity of $77 \%$ and specificity of $79 \%$ when dealing with four classes using OASIS dataset.

Behashti et al.,[5] presented an automatic computer-aided diagnosis(CAD) system for detection of Alzheimer's disease using structural MRI dataset from ADNI. The proposed method consists four stages. First, local and global difference in grey matter (GM) of AD patient and GM of normal healthy patient. Second, the voxel intensity values of the VOIs are extracted as raw feature. Third, the raw features are ranked by using seven-feature ranking methods. The feature with higher score value are more discriminative. Fourth, the Support Vector Machine (SVM) classifier used for classification. The classification accuracy of this proposed method for diagnosis of $\mathrm{AD}$ is up to $92.48 \%$ using sMRI data from state-of-the art databank ADNI.

We used state-of-the-art resting state functional magnetic resonance imaging (rs-fMRI) dataset from OASIS (Open Access Series of Imaging Studies). Dataset consists MRI slices of 416 subjects. We used MATLAB for image pre-processing steps- Realignment, Normalization, Segmentation, Outlier Detection, De-noising and smoothing. We got features of fMRI image to draw and analyse the distorted brain network of Alzheimer's Patient and the difference from the normal patient's brain network. We have deployed efficient intelligent computing methods in MATLAB for classification and diagnosis of Alzheimer's disease and generate a comparative study of accuracy obtained by these methods. We have used classifiers respectively Support Vector Machine (SVM), K-Nearest Neighbour (KNN) classifier and Naïve based Classifier. To 
further increase the accuracy, we used most relevant features using feature reduction principal component analysis (PCA) method and feature selection Maximum relevance minimum redundancy (MRMR) method. The proposed method achieved high accuracy via using 10-fold cross validation technique.

The comparative analysis of literature can be summarized by the below table.

Table-1: Comparative analysis of literature survey

\begin{tabular}{|l|l|l|}
\hline Paper & Methods & Results \\
\hline $\begin{array}{l}\text { Resting Brain Connectivity: } \\
\text { Changes during the progress } \\
\text { of Alzheimer Disease }\end{array}$ & $\begin{array}{l}\text { Temporal correlation method used to } \\
\text { obtain PCC connectivity maps. }\end{array}$ & $\begin{array}{l}\text { Functional connectivity } \\
\text { between the PCC and a set of } \\
\text { regions }\end{array}$ \\
\hline $\begin{array}{l}\text { Structural MRI-based } \\
\text { detection of Alzheimer's } \\
\text { disease using feature ranking } \\
\text { and classification error }\end{array}$ & CAD system & $\begin{array}{l}\text { Classification accuracy of the } \\
\text { proposed automatic system for } \\
\text { the diagnosis of AD is up to } \\
92.48 \% \text { using the sMRI data }\end{array}$ \\
\hline $\begin{array}{l}\text { Classification of Alzheimer } \\
\text { Diagnosis from ADNI } \\
\text { Plasma Biomarker Data }\end{array}$ & $\begin{array}{l}\text { five conventional classification } \\
\text { algorithms: libSVM }\end{array}$ & $\begin{array}{l}\text { The accuracy is 86\% for the } \\
\text { ensemble. }\end{array}$ \\
\hline $\begin{array}{l}\text { Classification of Alzheimer's } \\
\text { Disease Using fMRI Data } \\
\text { and Deep Learning } \\
\begin{array}{l}\text { Convolutional Neural } \\
\text { Networks }\end{array}\end{array}$ & Deep learning, CNN & Accuracy of 82\% \\
\hline $\begin{array}{l}\text { A novel method and software } \\
\text { for automatically classifying } \\
\text { Alzheimer's disease patients } \\
\text { by magnetic resonance } \\
\text { imaging analysis }\end{array}$ & $\begin{array}{l}\text { Computer vision method, called } \\
\text { Oriented FAST and Rotated BRIEF }\end{array}$ & Accuracy of 79\% \\
\hline $\begin{array}{l}\text { Brain connectivity and novel } \\
\text { network measures for } \\
\text { Alzheimer's disease } \\
\text { classification }\end{array}$ & SVM & $\begin{array}{l}\text { Brain network analysis of } \\
\text { pegree and betweenness } \\
\text { parameter }\end{array}$ \\
\hline
\end{tabular}

The paper's organization is as follows, Apart from introduction, Section II deals with problem description, section III deals with proposed methods including data acquisition, data preprocessing, proposed algorithm, section IV deals with results and discussion and section V represents conclusion.

\section{PROBLEM DESCRIPTION}

Early diagnosis of Alzheimer's disease (AD) via medical assessment is very costly and time consuming. The purpose of this paper is to propose a computational model which can differentiate the Alzheimer's disease patient and normal patient dataset. We have resting state 
functional magnetic resonance imaging (rs-fMRI) dataset from OASIS as input for computational models.

\section{PROPOSED METHOD}

\subsection{Data Acquisition}

Resting state functional magnetic resonance images (rs-fMRI) were obtained from state-of-the-art Open Access Series of Imaging Studies (OASIS) from (http://www.oasis-brains.org/). OASIS provide brain imaging data that are freely available for data analysis. The dataset consists of cross sectional images of 416 subjects having age 18 to 96 and covers all stages of AD. Additionally, images from a subsequent scan session after a short delay (less than 90 days) are also included as a means of assessing acquisition reliability, for 20 of the non-demented subjects. OASIS dataset also contains demographic, clinical, and derived anatomic measures located in the spreadsheets files (oasis_cross-sectional.xls and oasis_cross-sectional.csv). We used .nii format MRIs of all the subjects as our input dataset.

\subsection{Data Pre-processing}

Each subject has multiple slices in their MRI. The total number of slices in each subject's MRI is equal to 198. We applied functional Realignment for subject motion estimation and correction. After that, we applied translation of functional centre to $(0,0,0)$. Functional Slice-timing correction. MRIs are usually measured using 2D imaging methods repeatedly and this technique can result in a temporal offset difference between slices. We have applied slice-timing correction to mitigate the offset.

MRIs often contain unusual and dissimilar observations also called as outliers. This can happen for numerous reasons, for example, data acquisition, pre-processing artefacts, resulting from variance between large natural inter-subjects. But homogeneous samples are assumed in all of the statistical procedure. We have already centred the data using translation and we can use outlier detection to remove the dissimilarities.

To achieve simultaneous Grey/White/CSF segmentation, we applied functional direct segmentation and MNI Normalisation.

De-noising is also done using linear regression and band-pass filtering to remove physiological, unwanted motion, and other artefactual effects from the BOLD signal before computing connectivity measures. By default the system will start with three different sources of possible confounders: 1) BOLD signal from the white matter and CSF masks (5 dimensions each); 2) any previously-defined within-subject covariate (realignment and scrubbing parameters); and 3) the main condition effects (condition blocks convolved with hrf). For each of the selected possible confounds you may change the number of dimensions (specifying how many temporal components are being used), and the derivatives order (specifying how many successive orders of temporal derivatives are included in the model). For example, the realignment confound (derived from the estimated subject motion parameters) is defined by default by 6 dimensions. You can change the derivative order to 1 indicating that in addition the first-order temporal derivative of the motion parameters should also be used as covariates. Similarly, the White Matter confound is 
defined by default by 5 dimensions and 0 derivative order (indicating that 5 PCA temporal components are being used, with not additional temporal derivative terms).

\subsection{Computing Methods}

After the data pre-processing step, we use measures of efficiency, centrality, and cost/degree, associated with an ROI-to-ROI connectivity network built after second level analysis. After this, we use some popular machine learning models to build our classifier models to discriminate between Alzheimer's and non-Alzheimer's patients. We have 165 regions on brain network for each patient. We use degrees of each region node as our parameters for training the classifiers.

\subsubsection{Naive Bayes}

The naive Bayes classifier applies to learning tasks where each instance $\boldsymbol{x}$ is described by a conjunction of attribute values and where the target function $\mathrm{f}(\boldsymbol{x})$ can take on any value from some finite set V. A set of training examples of the target function is provided, and a new instance is presented, described by the tuple of attribute values $\left(a_{1}, a_{2} \ldots a_{n)}, a_{1}, a_{2} \ldots a_{n)}\right.$ The learner is asked to predict the target value, or classification, for this new instance.

\subsubsection{Support Vector Machine (SVM)}

Support vector machine is depends on pre-processing the data to higher dimension patterns rather than original feature space. The support vectors are the sample that are highly difficult for classification and the also define the hyperplane which should be optimally separated. The SVM finds the best classification function with largest margin of hyperplane separation between the two classes.

$$
L_{p}=\frac{1}{2}|| \vec{w} \|-\sum_{i=1}^{t} a_{i} y_{i}\left(\vec{w} \cdot \overrightarrow{x_{i}}+b\right)+\sum_{i=1}^{t} a_{i}
$$

Where $L_{p}$ is called the Lagrange, $\mathrm{t}$ is the number of training samples, and $a_{i}$ are the Lagrange multipliers, $\mathrm{b}$ is a constant and $\mathrm{w}$ is the vector that defines the hyperplane.

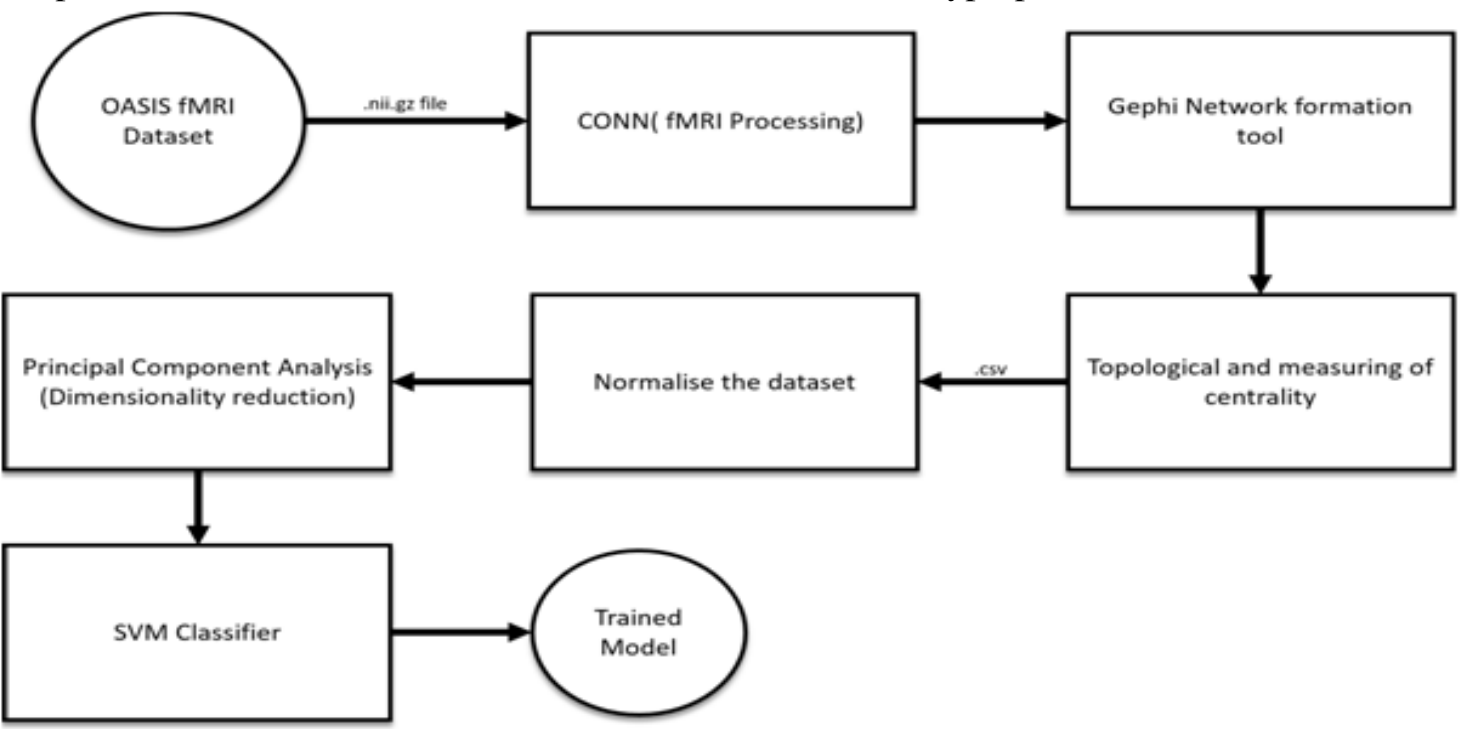

Figure 1. Flowchart of proposed model 


\subsubsection{K- Nearest Neighbour Classifier (KNN)}

K-Nearest Neighbour is one of the most basic yet essential classification algorithms in Machine Learning. It is widely disposable in real-life scenarios since it is non-parametric, meaning, it does not make any underlying assumptions about the distribution of data (as opposed to other algorithms such as GMM which assume a Gaussian distribution of the given data). In KNN we are given some prior data (also called training data), which classifies coordinates into groups identified by an attribute.

When a unknown tuple, a k-nearest neighbour classifier searches the pattern space for the $\mathrm{k}$ training tuples that are closest to the unknown tuple. The $\mathrm{k}$ training tuples are the $\mathrm{k}$ nearest neighbour of the unknown tuple.

\subsubsection{Principle Component Analysis (PCA) feature dimensionality reduction Method}

PCA is unsupervised feature transformation and linear projection method as vector projection proceeds without any knowledge of their class labels and reveals hidden information by maximizing the variance. An N-dimensional weighted feature $x_{i}(i=1,2,3 \ldots \ldots, m, N<m)$ was projected on the Eigenvectors of its covariance matrix and transformed matrix (v), the features of Eigenvalues greater than ' 1 ' is selected and tends to form a subset of 5 uncorrelated features. By PCA dimensionality is reduced. Few PCs can capture variances of the data. PCs are uncorrelated and ordered. We expect that cluster structure in the original dataset can be extracted from first few PCs.

$\mathrm{X}$ represents the original data matrix; $Y=\left(y_{1} \ldots \ldots, y_{n}\right), y_{i}=x_{i}-\bar{x}_{i}$ represents the centred data matrix where $\bar{x}-\sum_{i} x_{i} / n$. The covariance matrix (ignoring the factor $1 / \mathrm{n}$ ) is $\sum_{i}\left(x_{i}-\bar{x}\right)\left(x_{i}-\bar{x}\right)^{T}=Y Y^{T}$. Principal directions $u_{k}$ and principal components $v_{k}$ are eigenvectors satisfying $Y Y^{T} u_{k}=\lambda_{k} u_{k}, Y Y^{T} v_{k}=\lambda_{k} v_{k}, v_{k}=Y^{T} u_{k} / \lambda^{1 / 2}$

\subsubsection{MRMR Features selection method}

Minimal Redundancy Maximum Relevance is a feature selection method. Maximum Relevance means that selecting the features which has highest relevance with target class, based on mutual information, f-test And minimal redundancy means that selected features are correlated with each other and that features are cover narrow regions in the space.

Maximize Relevance:

$$
\max V_{I}, V_{I}=\frac{1}{|S|} \sum_{i \in S} I(h, i)
$$

Where $s$ is the set of features, $I(i, j)$ is mutual information between feature $i$ and $j$.

Minimal Redundancy:

$$
\min W_{I}, W_{I}=\frac{1}{|S|^{2}} \sum_{i, j \in S} I(i, j)
$$




\subsection{Pseudo-code for the proposed model}

/* Performance are measured by k-fold Cross Validation, where $\mathrm{k}=10 * /$

Begin:

- fMRI processing steps- Realignment, Normalization, Segmentation, Outlier Detection, De-noising and smoothing $->$ Brain Network Topological and centrality measurement

- $\quad$ For $\mathrm{i}=1$ to $\mathrm{k}$ :

- $\quad$ EndFor;

○ k-1 subset $\rightarrow$ Training set;

○ Remaining subset $->$ Testing set;

- Rank features technique, $\mathrm{mRMR}$;

- Dimension reduction technique, PCA

- Train SVM, KNN and Naïve bayes classifier on reduced space training dataset using different size of feature subset;

$\circ$ Test the trained SVM, KNN and Naïve bayes models on Testing set;

- Calculate the average classification accuracy of SVM, KNN and Naïve bayes over $i^{\text {th }}$ testing set;

End.

\section{RESULTS}

As per workflow diagram of proposed model, first we generate brain graph network of more concerned Region of Interest (ROI) of brain related to Alzheimer disease. We used MATLAB for image pre-processing steps- Realignment, Normalization, Segmentation, Outlier Detection, Denoising and smoothing. We got features of fMRI image to draw and analyze the distorted brain network of Alzheimer's Patient and the difference from the normal patient's brain network. Now, using gephi tool, we generate .csv file of topological measures and centrality. As per paper [3], degree and betweenness are two important parameters for classifier model. But in our method we used other parameters average path length, clustering coefficient, cost, local and global efficiency too with degree and betweenness. Then we used PCA for dimensionality reduction and MRMR for relevant feature selection. In compare to paper [3], we got increased accuracy $95 \%$ using SVM, 95\% using KNN and 90\% from Naïve bayes classifier.

Table-2: Sensitivity \& Specificity

\begin{tabular}{|l|c|c|c|c|}
\hline Method & True Positive & False Positive & True Negative & False Negative \\
\hline SVM & 10 & 1 & 9 & 0 \\
\hline Naive Bayesian & 10 & 2 & 8 & 0 \\
\hline k-nearest neighbour & 10 & 1 & 9 & 0 \\
\hline
\end{tabular}




\section{SVM}

Initially the features that are extracted from the MRI image are used to train the SVM classifier. In training phase, the model is built while in testing phase, the model is validated. There is only one type of classification performed in which the features are fed to SVM which creates models numbered -1 for Alzheimer's disease and +1 for normal person.

In the testing period, all of the features are input into SVM and distance between each of the vector and hyperplane is calculated. We achieved a true positive rate of $100 \%$ and false positive rate of $10 \%$ and the accuracy of our model is $95 \%$.

\section{Naive Bayesian}

Naive Bayesian depends upon the selection of training data for increasing the accuracy of the classifier. Partitioned vectors of processed MRI's features are used to test and train the Naive Bayesian classifier. This also uses the same class models as SVM numbered +1 and -1 for normal person and Alzheimer's disease respectively.

In the testing phase, 20 vectors are validated out of which we received a true positive rate of $100 \%$ and a false positive rate of $20 \%$ which gives a total accuracy of $90 \%$.

\section{K-nearest Neighbour}

In the testing phase, 20 vectors are validated out of which we received a true positive rate of $100 \%$ and a false positive rate of $20 \%$ which gives a total accuracy of $90 \%$.

In compare to other methods proposed by authors [3],[4],[14],[15], we get more accurate classifier model. For the relative comparison refer to table-1 and literature survey.
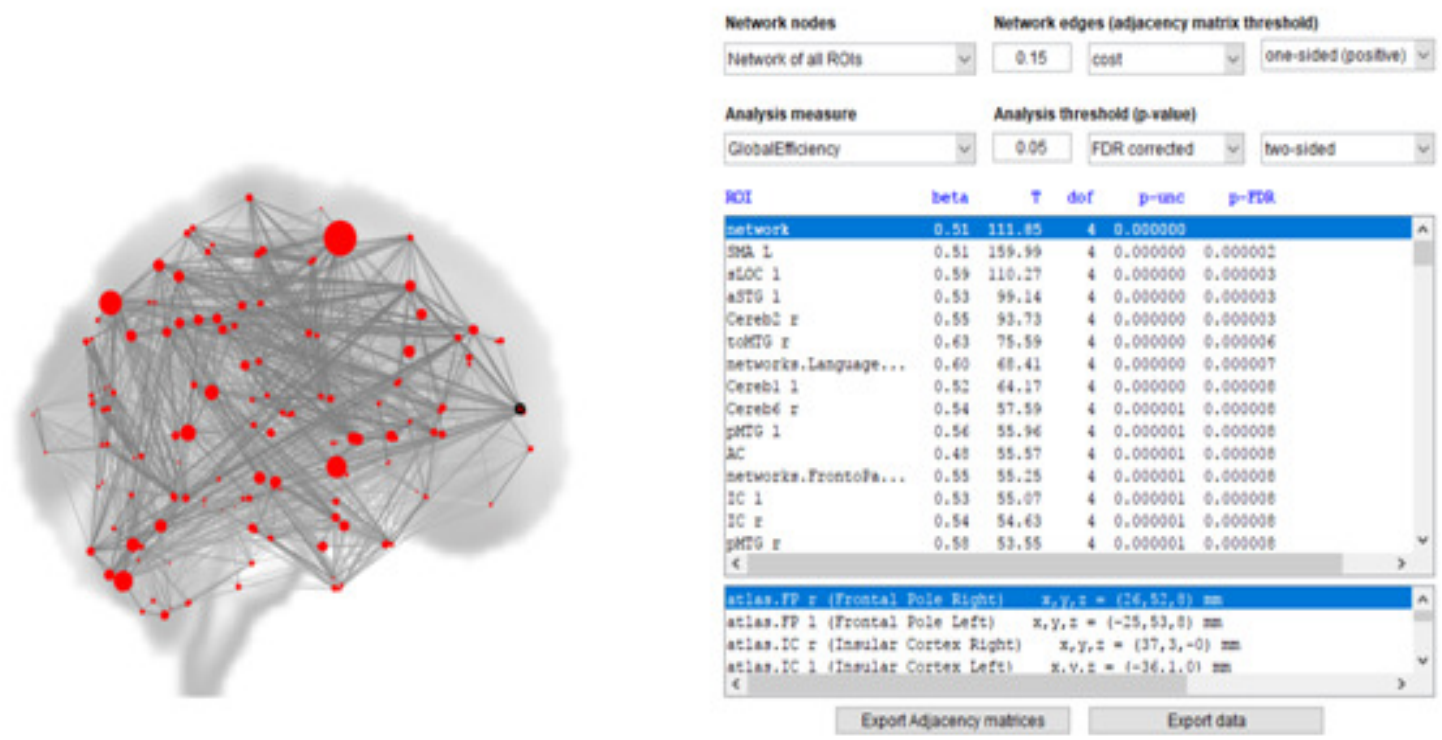

Figure 2. Brain network generated from OASIS AD data 


\section{DISCUSSION \& CONCLUSION}

Functional magnetic resonance imaging or functional MRI (fMRI) measures brain activity by detecting changes associated with blood flow. Using BOLD (Blood Oxygen level dependent) technique accurate brain data can be captured as fMRI image. We use state-of-the art database OASIS for this purpose. By proper processing of fMRI images and pre-processing approaches we get brain connectivity network. Alzheimer or any other neurodegenerative diseases can be captured by analysing this brain connectivity network. . The grey matter activity of Alzheimer disease patient is not as high as a non-Alzheimer patient. The grey matter stands for oxygen level in the brain. It is intuitive that oxygen level in Alzheimer's disease patient is lower than a normal brain. Oxygen level also shows the amount of activity being done in the brain, so a higher oxygen level brain means active brain. In our proposed method, finally using SVM, KNN and Naïve bayes, we get success in making classifier model with quite good accuracy of $95 \%, 95 \%$ and $90 \%$. In our proposed method we focus on brain connectivity disturbance due to Alzheimer's disease. There are other areas also, which got affected by neuro diseases like blood cells, proteinprotein interaction, gene-gene interaction etc. We will try to all incorporate all effective regions during design of classifier model. Other than organic areas like brain network, blood cells, protein and genes, non-organic data like Demographic, clinical, and derived anatomic measures can also be used for classifier model. In this proposed method we used OASIS fMRI dataset of Alzheimer's disease and used SVM classifier model to classify neuro disease. In future we will use other dataset like ADNI and composition of other classifier models like libSVM(an ensemble of five conventional algorithms) as described in paper[14].

\section{REFERENCES}

[1] Biju, K. S., S. S. Alfa, Kavya Lal, Alvia Antony, and M. KurupAkhil. "Alzheimer's Detection Based on Segmentation of MRI Image." Procedia Computer Science 115 (2017): 474-481.

[2] Sarraf, Saman, and GhassemTofighi. "Classification of alzheimer's disease using fmri data and deep learning convolutional neural networks." arXiv preprint arXiv:1603.08631 (2016).

[3] Goñi, Joaquín, Francisco J. Esteban, Nieves Vélez de Mendizábal, Jorge Sepulcre, Sergio ArdanzaTrevijano, Ion Agirrezabal, and Pablo Villoslada. "A computational analysis of protein-protein interaction networks in neurodegenerative diseases." BMC systems biology 2, no. 1 (2008): 52.

[4] Zhao, Jinying, Yun Zhu, Jingyun Yang, Lin Li, Hao Wu, Philip L. De Jager, Peng Jin, and David A. Bennett. "A genome-wide profiling of brain DNA hydroxymethylation in Alzheimer's disease." Alzheimer's \& dementia: the journal of the Alzheimer's Association 13, no. 6 (2017): 674-688.

[5] Beheshti, Iman, Hasan Demirel, FarnazFarokhian, Chunlan Yang, Hiroshi Matsuda, and Alzheimer's Disease Neuroimaging Initiative. "Structural MRI-based detection of Alzheimer's disease using feature ranking and classification error." Computer methods and programs in biomedicine 137 (2016): 177-193.

[6] Zhang, Hong-Ying, Shi-Jie Wang, Bin Liu, Zhan-Long Ma, Ming Yang, Zhi-Jun Zhang, and Gao-Jun Teng. "Resting brain connectivity: changes during the progress of Alzheimer disease." Radiology 256, no. 2 (2010): 598-606.

[7] Previtali, Fabio, Paola Bertolazzi, Giovanni Felici, and Emanuel Weitschek. "A novel method and software for automatically classifying Alzheimer's disease patients by magnetic resonance imaging analysis." Computer methods and programs in biomedicine 143 (2017): 89-95. 
[8] Prasad, Gautam, Shantanu H. Joshi, Talia M. Nir, Arthur W. Toga, and Paul M. Thompson. "Brain connectivity and novel network measures for Alzheimer's disease classification." Neurobiology of aging 36 (2015): S121-S131.

[9] Wang, Hong, Wenwen Chang, and Chi Zhang. "Functional brain network and multichannel analysis for the P300-based brain computer interface system of lying detection." Expert Systems with Applications 53 (2016): 117-128.

[10] Li, Kaiming, Lei Guo, JingxinNie, Gang Li, and Tianming Liu. "Review of methods for functional brain connectivity detection using fMRI." Computerized Medical Imaging and Graphics 33, no. 2 (2009): 131-139.

[11] Sheline, Yvette I., and Marcus E. Raichle. "Resting state functional connectivity in preclinical Alzheimer's disease." Biological psychiatry 74, no. 5 (2013): 340-347.

[12] Plant, Claudia, Christian Sorg, Valentin Riedl, and AfraWohlschläger. "Homogeneity-based feature extraction for classification of early-stage alzheimer's disease from functional magnetic resonance images." In Proceedings of the 2011 workshop on Data mining for medicine and healthcare, pp. 3341. ACM, 2011.

[13] Mo, Jue, Sana Siddiqui, Stuart Maudsley, Huey Cheung, Bronwen Martin, and Calvin A. Johnson. "Classification of Alzheimer Diagnosis from ADNI Plasma Biomarker Data." In Proceedings of the International Conference on Bioinformatics, Computational Biology and Biomedical Informatics, $p$. 569. ACM, 2013.

[14] Biswal, Bharat, F. ZerrinYetkin, Victor M. Haughton, and James S. Hyde. "Functional connectivity in the motor cortex of resting human brain using echo-planar mri." Magnetic resonance in medicine 34, no. 4 (1995): 537-541.

[15] Li, Shi-Jiang, Bharat Biswal, Zhu Li, Robert Risinger, Charles Rainey, Jung-Ki Cho, Betty Jo Salmeron, and Elliot A. Stein. "Cocaine administration decreases functional connectivity in human primary visual and motor cortex as detected by functional MRI." Magnetic Resonance in Medicine 43, no. 1 (2000): 45-51.

\section{AUTHORS}

Rishi Yadav - Final year Integrated dual degree $($ Btech + Mtech) student in Computer Science and Engineering at Indian Institute of Technology, BHU, Varanasi, India.

Email- rishi.yadav.cse13@iitbhu.ac.in

Ankit Gautam - Final year Integrated dual degree (Btech + Mtech) student in Computer Science and Engineering at Indian Institute of Technology, BHU, Varanasi, India.

Email-ankit.gautam.cse13@iitbhu.ac.in

Ravi Bhushan Mishra- Professor in Computer Science and Engineering at Indian Institute of Technology, BHU, Varanasi, India.

Email- mishraravi.cse@itbhu.ac.in 